\title{
On the Mongolian verb of motion yav- 'to go, to travel, to leave'
}

\author{
Veronika Zikmundova \\ Charles University, Prague
}

\section{Introduction}

Since the 1970s, verbs of motion as an important part of the vocabularies of most languages have been extensively studied by cognitive linguists, in particular, Talmy (1975, 1985, 2000), Levin and Rappaport Hovav (1992), Slobin (1996), and others. ${ }^{1}$ Based on Talmy's terminological apparatus (Figure, Path, Manner, Ground, etc.), a series of studies have featured the "universals" of the usage of verbs of motion in many languages.

This cross-linguistic research has shown that languages tend to fall into two major classes in terms of how they express meaning connected to motion in space these are the "verb-framed" vs. "satellite-framed" languages. According to this classification, the Altaic languages generally belong to the former class. Recently, Nakazawa $(2007,2009)$ has focused on verbs of motion in Mongolian. Some recent studies have emphasized the necessity of both more focused and intense methods of apprehending the verbal systems of motion in various languages (Word-Allbritton 2004: 9-10).

It is my conviction that that the descriptive approach to particular languages, based on careful examination of larger samples of language data, contributes important information to the pragmatics of the studied languages. The description of their semantics, including the grammaticalized and figurative usage of particular verbs of motion, may become one of the clues to comprehending the "linguistic mind" and the ways in which particular languages view and reflect on the world.

The present paper continues a discussion of the topic of verbs of motion in Mongolian and Sibe, which I described in previous articles (Zikmundova 2010,

\footnotetext{
${ }^{1}$ See the bibliography by Slobin and Matsumoto at http://www.lit.kobe-u.ac.jp/ yomatsum/motionbiblio1.pdf.
} 
2011), and continues the examination of the Sibe verb yaf- (2013). The general translation of the Sibe verb yaf-and the Khalkha Mongolian verb yav-are largely similar, covering the meanings 'to go', 'to travel', 'to depart', and in a more the abstract or figurative sense referring to various aspects of existing.

The verbs of motion which I examined in previous papers (Sibe gen- 'to go there, to go' and $j i$ - 'to come here', Khalkha Mongolian $o c^{-}$- 'to go there', ir- 'to come here') form a part of a largely coherent system of verbs of motion consisting of antonymical pairs with distinct spatial semantics, which is typical of Altaic languages in general. However, the verb with the general meaning of motion, examined in the present article, lacks some characteristics of this system, tending rather to resemble the usage of verbs of motion in the "satellite-framed" languages. In particular, while it is difficult to establish an accurate English translation of the verbs 'to come here' or 'to go there' which would encompass the entire range of meanings, both the Sibe verb yaf-and the Mongolian verb yav-, including much of their figurative meanings, are aptly translated with the English verb 'to go'. An important difference between English on the one hand and both Sibe and Mongolian on the other lies in the emphasis on motion as the basic aspect of life, which is mirrored in the semantics of both of these verbs.

In the present paper I examine the semantics of the Khalkha Mongolian verb $y a v$-, based on examples from Modern Khalkha Mongolian. The analyzed data come from various sources: folktales and proverbs retrieved from the Database of Mongolian folklore texts at http://www.signeta.cz/textsearch/, interviews recorded by V. Kapišovská in the Khentii Province of Mongolia in 2014, utterances noted during everyday conversations in 2015, and texts and conversations available on the Internet. The examples are roughly divided into "literal" and "figurative" usage and further classified into tentative groups according to the manner of usage.

\section{The verb yav-expressing the literal meaning of 'motion'}

The basic semantics of the verb yav-is connected to the process of motion. Within its range of meanings, the emphasis on 'leaving, or setting off [in motion]' and 'to undertaking a journey' seem to be especially frequent.

\subsection{The verb yav-designating the process of motion}

The general meaning of motion in space seems to be the basic and most important component within the semantics of the Mongolian verb yav-. In this literal meaning 
the agent is usually either an animate being, or an object for which such motion is a typical action. In this type of usage spatial determination may be either present (sentences 1 and 2) or absent (sentence 3). Unlike in other Mongolian verbs of motion, however, here the semantic emphasis is on the motion itself rather than on its goal or direction.

(1)

$\begin{array}{lllll}\text { Нөгөө хүн } & \text { тэр } & \text { явж } & \text { байна. } \\ \text { nögӧ xün } & \text { ter } & \text { yav-ž } & \text { bai-na. } \\ \text { that* } & \text { person } & \text { that } & \text { go-CI } & \text { be-IMPRF }\end{array}$

(*in reference to a previously mentioned fact)

'That [previously mentioned] person is walking/riding over there.'

(2)

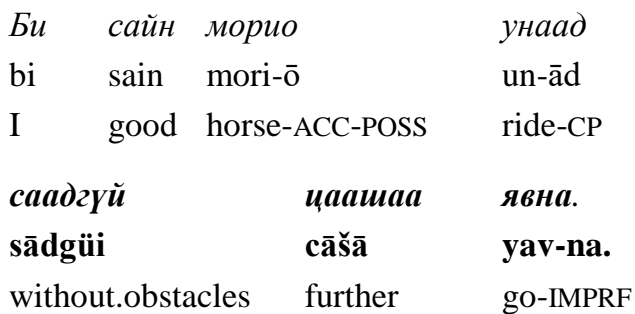

'I will ride [on] my good horse and proceed smoothly.'

(3)

\begin{tabular}{|c|c|c|c|}
\hline Улаан иирх & явна. & $Y_{\text {Үнийг }}$ & ална \\
\hline širx & yav-na. & Ün-1̄g & al-na \\
\hline louse & go-IMPRF & this-ACC & kill-IMPRF \\
\hline
\end{tabular}

'A red louse is creeping [towards the location of the speaker]. Should I kill it?'

\subsection{The verb yav- - expressing the meaning of a journey with a specific purpose}

While designating the process of motion, many of the examined examples can also be interpreted as referring to a whole journey with a certain goal and purpose, which may be either overtly expressed (4)-(7), or understood from the context (9). When the goal of motion is defined spatially, it is mostly in the lative case (4), (9). In

\footnotetext{
${ }^{2}$ The abbreviations in the interlinear glosses follow, for the most part, the list of standard abbreviations available at https://www.eva.mpg.de/lingua/pdf/Glossing-Rules.pdf (Leipzig glossing rules). The rest is abbreviated from grammatical terms used in Vacek and Luvsandorj (2004).
} 
colloquial usage, however, the case suffix is often omitted (8). When the purpose of motion is expressed by a verb, the latter mostly has the form of a verbal noun in the instrumental case, which is a form typically used for the expression of purpose - (7), (9), or employs an analytic construction. When the purpose of motion is expressed by a noun, it usually stands in the dative-locative (6), (10).

$\begin{array}{lllll}\text { Ta } & \text { zадагшаа } & \text { явсан } & \text { юм } & \text { yy? } \\ \text { ta } & \text { gada-gšā } & \text { yav-san } & \text { yum } & \text { uu? } \\ \text { you } & \text { outside-LAT } & \text { go-NP } & \text { PTC } & \text { Q } \\ \text { 'Oh, you have gone elsewhere? [You are not here?]' }\end{array}$

(5)

$\begin{array}{lll}\text { Нараа эгч } & \text { Монгол } & \text { явсан. } \\ \text { Narā egс̌ } & \text { Mongol } & \text { yav-san. } \\ \text { naraa elder.sister } & \text { Mongolia } & \text { go-NP }\end{array}$

'Aunt Naraa went to Mongolia/ is in Mongolia.'

(6) Арван гурван жилийн анд явсан аав

arvan gurvan žil-īn an-d yav-san āv

ten three year-GEN hunt-DL go-NP father

хаан чинь хурээд ирэв.

xān čin' xür-ēd ir-ev.

where POSS.2SG arrive-CP come-PRET.PERF

'Your father, who left for a hunt of thirteen years, has come back.'

(7) Гадаадад сурахаар явах хүмүУст

gadāda-d sura-x-ār yava-x xümüs-t

abroad-DL study-NF-INS go-NF people-DL

банкны батлагаа гаргана.

bankn-ï batlagā garga-na.

bank-GEN certificate issue-IMPRF

'We issue a bank certificate for those who go to study abroad.'

(8) Аав аа, аав аа! Ямар мориороо явах

$\bar{a} v-\bar{a}, \quad \bar{a} v-\bar{a} ! \quad$ Yamar mori-or-ō yava-x ve

father-VOC father-VOC what horse-INS-POSS.REF go-NF Q

'Father, with which horse should I go?' 
(9)

\begin{tabular}{|c|c|c|c|c|}
\hline Нөхөр & & Баянхонгор & аймаг руу & ажил \\
\hline nöxör & & Bayanxongor & aimag-rū & ažil \\
\hline husband $\mathrm{P}$ & S1PL.GEN & Bayankhongor & aimag-LAT & work \\
\hline хийхээр & яваад & эзгүй & & \\
\hline $\mathbf{x} \overline{\mathbf{1}}-\mathbf{x}-\bar{e} \mathbf{r}$ & yav-ād & ezgüi & & \\
\hline $0-N F-I N S$ & go-CP & absent & & \\
\hline
\end{tabular}

'My husband went to Bayankhongor aimag to work and was not at home.'

(10) Ухнанд явсан хүY ямаа иииглэсэн хойно ирэв.

uxnan-d yav-san $x \bar{u}$ yamā išigle-sen xoino ire-v.

ram-DL go-NP boy goat give.birth-NP after come-PRET.PERF

'The boy who went to fetch the ram returned after the goats gave birth.'

\subsection{The verb yav-expressing the meaning 'to set oneself in motion, to leave'}

In everyday colloquial usage, the verb yav-is frequently used in connection with leaving a certain site, setting off, etc.
(11) $3 а$ одоо явичгаая!
za odō yav-cgā-ya!
PTC now go-VP-VOL
'So, let's go now!'
(12) Ээж явсан юм уу?
ēž yav-san yum $\bar{u}$ ?

mother go-NP PTC Q

'Has Mother left?'
(13) Явахаасаа өмнө надаас юу хуссэнээ ав! yava-x-ās-ā ömnö nad-ās yü xüs-sen-ē av!
go-NF-ABL-POSS before 1SG-ABL what wish-NP-ACC-POSS take-IMP 'Before you leave, take anything you wish from me.'
(14) Явна гвна ямаань мах барав...
yav-na yav-na ge-ž yamān-ï max bara-v.
go-IMPRF go-IMPRF say-CI goat-GEN meat finish-PRET.PERF

'Saying "I am leaving," he finished off the meat of an [entire] goat.' 
(15)

$\begin{array}{lllllr}\text { Тэр } & \text { охин } & \text { урьд } & \text { нь } & \text { гэрээсээ } & \text { хоёр, } \\ \text { ter } & \text { oxin } & \text { ur'd } & \text { n' } & \text { ger-ēs-ē } & \text { xoyor, } \\ \text { that } & \text { girl } & \text { before } & \text { POSs3sG } & \text { home-ABL-POSS two }\end{array}$

гурван удаа явж байсан гэнэ.

gurvan udā yav-ž bai-san ge-ne.

three time go-CI be-NP say-IMPRF

'They say that formerly this girl had left home two or three times.'

(16)

$\begin{array}{lllll}\text { Дахиж } & \text { гэрээсээ } & \text { хэзээ } & 4 & \text { явахгүй. } \\ \text { daxiž } & \text { ger-ēs-ē } & \text { xezē } & \check{c} & \text { yava-X-güi. } \\ \text { again } & \text { home-ABL-POSS } & \text { when } & \text { PTC } & \text { go-NF-NEG }\end{array}$

'I will never leave home again.'

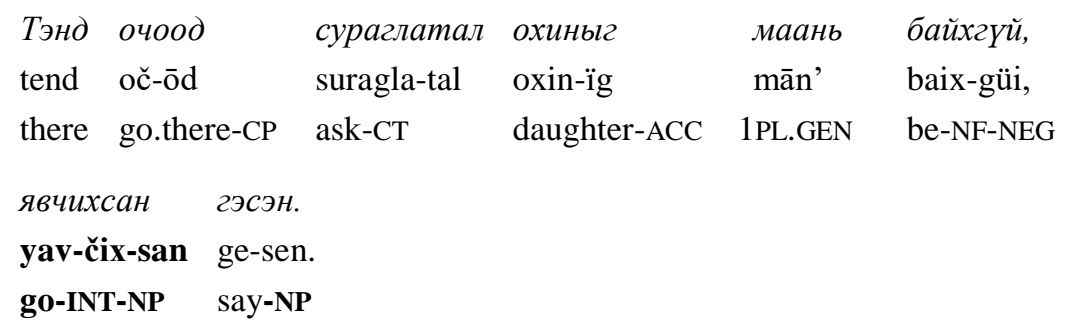

'When we went there to ask, we were told our daughter wasn't there; she had left.'

\section{Modal and grammatical usage of the verb yav-}

\subsection{The verb yav-expressing a modality of continuous motion}

A frequent type of phrase is one where the verb yav-is preceded by another verb of motion in the form of an imperfective converb. In such phrases, the function of the verb yav- may be interpreted as modal, foregrounding the notion of continuous motion. This kind of usage is typical for dependent phrases with a temporal meaning (sentences 20-22).

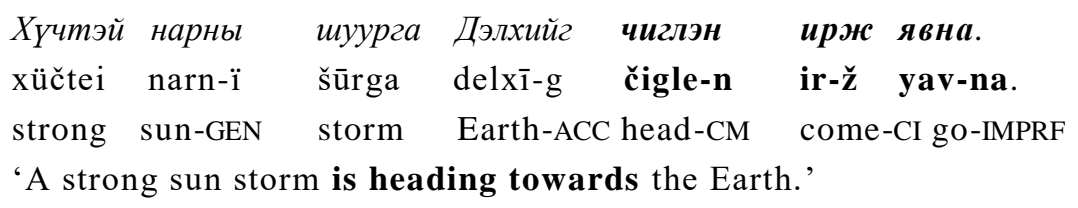


(19)

\begin{tabular}{|c|c|c|c|c|c|}
\hline Хоёр & залуу & ц̧ирк & Узээд & zapu & явна. \\
\hline yor & zalū & cirk & üz-ēd & gar-č & yav-na. \\
\hline wo & youngster & circu & see-CP & go.out-CI & go-IMPRF \\
\hline
\end{tabular}

'Two young men are leaving the circus after a performance.'

(20)

$\begin{array}{llll}\text { Уртын даваа } & \text { даваад } & \text { баруун } & \text { тийшээгээ } \\ \text { Urtïn davā } & \text { dav-ād } & \text { barūn } & \text { tī-šē-gēe } \\ \text { Urtyn pass } & \text { surmount-CP } & \text { west } & \text { there-LAT-POSS.REF }\end{array}$

буужс явахад ...

bū-ž $\quad$ yava-x-ad...

descend-CI go-NF-DL

'As they crossed the Urtyn Pass and were descending in a westerly direction...'

$\begin{array}{lllll}\text { Буцажж } & \text { явах } & \text { замдаа } & \text { осол } & \text { гаргасан. } \\ \text { buca-ž } & \text { yava-x } & \text { zam-d-aa } & \text { osol } & \text { gar-ga-san } \\ \text { Return-CI } & \text { go-NF } & \text { way-DL-POSS.REF } & \text { accident } & \text { go.out-CAUS-NP }\end{array}$

'On the way back, he caused a car accident.'

(22) Гэртээ

$$
\text { харьжс }
$$

явтал гудамжнь

ger-t-ē

xar'-ž

yav-tal

gudamžn-ï

home-DL- POSS.REF

return.home-CI

go-CT

treet-GEN

$\begin{array}{lllll}\text { өнцуөгт } & \text { гоё } & \text { бүсгуй } & \text { зогсохьгг } & \text { Үзээд } \\ \text { öncög-t } & \text { goуо } & \text { büsgüi } & \text { zogsox-їg } & \text { üz-ēd } \\ \text { corner-DL } & \text { beautiful } & \text { woman } & \text { stand-NF-ACC } & \text { see-CP }\end{array}$

'As he was returning home, he saw a beautiful woman standing on the corner of the street...'

(23)

$\begin{array}{llllll}\text { Гурван хүн } & \text { машинаар } & \text { чоно } & \text { хөөж } & \text { яваад } & \text { осолджэээ. } \\ \text { gurvan xün mašin-ār } & \text { čono } & \text { xō̄-ž } & \text { yav-ād } & \text { osold-žē. } \\ \text { three person car-INS } & \text { wolf } & \text { hunt-CI } & \text { go-CP } & \text { crash-PRET.IMP }\end{array}$
'Three people, hunting wolves while [driving] in the car, were in an accident.' 


\subsection{The verb yav-expressing a modality of intensity and change}

This modal usage is derived from the basic semantics of 'setting oneself in motion' or 'leaving'. In this type of phrases the verb yav-adds a modal colouring of an intensive and persistent change of state (Ex. 24-26), or of an intensive action (27).

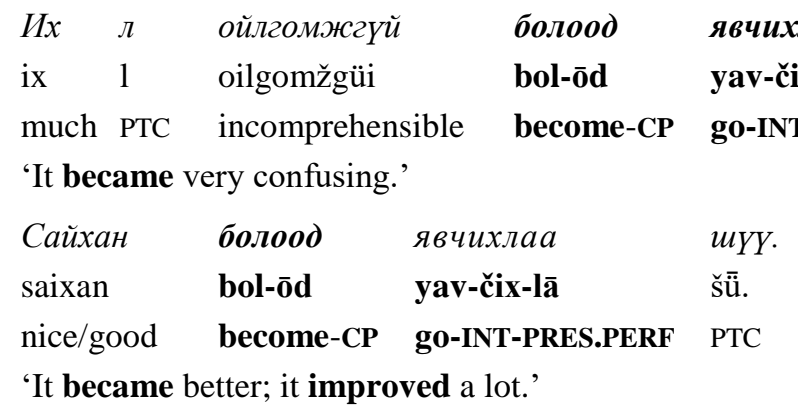

$\begin{array}{llll}\text { Мамины } & \text { наймаанд } & \text { яваад } & \text { амьдрал } \\ \text { mašin-ï } & \text { naimān-d } & \text { yav-ād } & \text { am'dral } \\ \text { car-GEN } & \text { business-DL } & \text { go-CP } & \text { life } \\ \text { 'nод' } & \text { хийгээд } & \text { явчихлаa. } & \\ \text { 'pod' } & \text { xīg-ēd } & \text { yav-čix-lā. } & \\ \text { 'pod' } & \text { do-CP } & \text { go-INT-PRES.PERF }\end{array}$

'After I [started] doing trade in buying and selling cars, my life improved.'

\begin{tabular}{|c|c|c|c|c|c|}
\hline $\begin{array}{l}\text { Унтаж } \\
\text { unta-ž } \\
\text { sleep-CI }\end{array}$ & $\begin{array}{l}\text { байтал } \\
\text { bai-tal } \\
\text { be-ст }\end{array}$ & $\begin{array}{l}\text { толгой } \\
\text { tolgoi } \\
\text { head }\end{array}$ & $\begin{array}{l}\partial э \ni p \\
\text { dēr } \\
\text { on }\end{array}$ & $\begin{array}{l}\text { минь } \\
\text { min' } \\
\text { POSS1SG }\end{array}$ & \\
\hline юм & таш баш & хийгээд & явчи. & лаа & $m Y Y$. \\
\hline yum & taš baš & xīg-ēd & yav-č & $x-l \bar{a}$ & šū̄. \\
\hline thing & bash & do-CP & go-IN & T-PRES.PERF & PTC \\
\hline
\end{tabular}

'When I was sleeping, something made a bumping noice above my head.'

\subsection{The verb yav-in auxiliary function}

A situation that describes two speakers encountering each in open space, when one or both speakers are in motion, employs the verb yav-as the final member of a verb phrase following other verbs which are non-motion verbs (28-31). In this kind of situation-typical in the life of nomadic herders - the verb yav- may possibly be interpreted as expressing a grammatical meaning very close to the auxiliary 
existential verb bai- 'to be.' In most of the examples below, the verb yav-could be replaced by bai- without any substantial change in the meaning of the sentence.
Барон Унгерний
тухай судлах
гээठ
яваа юм уу?
baron Ungern- $\overline{1}$ tuxai sudla-x
ge-ēd
yav-ā yum $\overline{\mathbf{u}}$ ?
Baron Ungern-GEN about research-NF say-CP
go-NI PTC $Q$

'So you are travelling with the purpose of studying Baron Ungern?'

$\begin{array}{llll}\text { Та нар } & \text { юунд } & \text { ява丿 хүмҮҮс } & \text { вэ? } \\ \text { Ta-nar } & \text { yūn-d } & \text { yav-ā xümüs } & \text { ve? } \\ \text { you-PL } & \text { what-DL } & \text { go-NI people } & \text { Q }\end{array}$

'What is the purpose of your journey?'

(30)

$\begin{array}{llll}\text { Намайг } & \text { алж } & \text { идэх } & \text { шахлаа. } \\ \text { Nama-ig } & \text { al-ž } & \text { ide-x } & \text { šax-lā. } \\ \text { 1SG-ACC } & \text { kill-CI } & \text { eat-NF } & \text { press-PRES.PERF }\end{array}$

$\begin{array}{lllll}\text { Тэгээд } & \text { би } & \text { ингэж } & \text { зугатаж⿻ } & \text { явна. } \\ \text { tegēd } & \text { bi } & \text { inge- } \check{\text { ž }} & \text { zugata-̌̌ } & \text { yav-na. } \\ \text { so } & \text { I } & \text { like.this } & \text { flee-CI } & \text { go-IMPRF }\end{array}$

'They almost killed me, so I am running like this.'

(31)

$\begin{array}{lllll}\text { Бар } & \text { чи } & \text { юундаа } & \text { ингэж } & u x \\ \text { bar } & \text { či } & \text { yūn-d-ā } & \text { ingež } & \text { ix } \\ \text { tiger } & \text { you } & \text { what-DL-POSS.REF } & \text { like.this } & \text { much }\end{array}$

$\begin{array}{ll}\text { сандарч } & \text { явна? } \\ \text { sandar-č } & \text { yav-na? }\end{array}$

be.anxious-CI go-IMPRF

'Why are you so anxious, tiger?'

$\begin{array}{lllll}\text { Судалгаa } & \text { хииж } & \text { явсан } & \text { ажилтньг } & \text { хутгалж } \\ \text { sudalgā } & \mathbf{x \overline { \mathbf { I } } - \mathbf { z }} & \mathbf{y a v - s a n} & \text { ažiltn-ïg } & \text { xutgal-ž } \\ \text { research } & \text { do-CI } & \text { go-NP } & \text { worker-ACC } & \text { stabb-CI }\end{array}$

$\begin{array}{llllll}\text { амь } & \text { насыг } & \text { нь } & \text { хохироосон } & \text { хэрэг } & \text { гарчээ. } \\ \text { am' } & \text { nas-ïg } & \text { n' } & \text { xохіrō-son } & \text { xereg } & \text { gar-čē. }\end{array}$

life age-ACC POSS3SG harm-NP matter go.out-PRET.IMP

'It happened that an employee was stabbed to death while doing field research.' 


$\begin{array}{ll}\text { YҮргээ } & \begin{array}{l}\text { гүйцэтгээ⿻ } \\ \text { güicetge-ž }\end{array} \\ \text { ürgē } & \text { fulfill-CI } \\ \text { task-POSS.REF } & \text { алджээ } \\ \text { aмиа } & \text { ald-žē } \\ \text { ami-a } & \text { lose-PRET.PERF }\end{array}$

'Two policemen lost their lives while in service.'

$\begin{array}{lllrlrl}\text { Ихэнхуээ } & \text { хоолыг } & \text { нь } & \text { дээрэмдээд } & \text { явсан } & \text { юм. } \\ \text { inenxdē } & \text { xōl-ïg- } & \text { n' } & \text { dēremd-ēd } & \text { yav-san } & \text { yum. } \\ \text { mostly } & \text { food-ACC } & \text { POSS3SG } & \text { rob-CP } & \text { go-NP } & \text { PTC }\end{array}$

'He was mainly stealing their food.'

\subsection{The verb yav- as a member of a complex descriptive expression}

The verb yav-, when forming part of a complex description of actions, shows lesser variability in its usage than other verbs of motion. This is due to its lack of a concrete deictic function.

\subsubsection{The verb yav- in initial phrasal position}

When placed in initial phrasal position, the verb yav- usually describes either a departure with consequent motion (35-38) or a process of motion (39-40). In the latter case, it often adds a modal colouring of slight expectation followed by surprise (39).

$\begin{array}{lllll}\text { Хөөш } & \text { чи } & \text { талханд } & \text { яваад } & \text { ир! } \\ \text { хӧš } & \text { či } & \text { talxan-d } & \text { yav-ād } & \text { ir! } \\ \text { hey } & \text { you } & \text { bread-DL } & \text { go-CP } & \text { come }\end{array}$

'Go buy bread, please!'

$\begin{array}{lllll}\text { Аав аa, } & \text { аав аa, } & \text { би } & \text { алтан } & \text { мөнгөн } \\ \overline{\mathrm{a}} \mathrm{v}-\overline{\mathrm{a}}, & \overline{\mathrm{a}} \mathrm{v}-\overline{\mathrm{a}}, & \text { bi } & \text { altan } & \text { möngön } \\ \text { father-VOC } & \text { father-VOC } & \mathrm{I} & \text { golden } & \text { silver } \\ \text { aргaйндаa } & & & \text { яваaд } & \text { ирьe! } \\ \text { argain-d-ā } & & & \text { yav-ād } & \text { ir-ye } \\ \text { playing.bone-DL-POSS.REF } & & \text { go-CP } & \text { come-VOL }\end{array}$


'Father, I will go fetch my golden and silver playing bones.'

(37)

$\begin{array}{llllll}\text { Би } & \text { өчигдөр } & \text { Хархорум хот } & \text { яваад } & \text { ирсэн. } \\ \mathrm{Bi} & \text { öčigdör } & \text { Xarxorum } & \text { xot } & \text { yav-ād } & \text { ir-sen. } \\ \text { I } & \text { yesterday } & \text { Kharkhorum city } & \text { go-CP } & \text { come-NP }\end{array}$

'Yesterday I paid a visit to Kharakhorum city.'

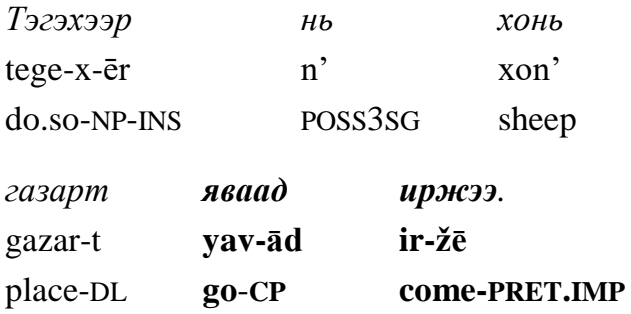

'And so he arrived at the place where the sheep had been before.'

$\begin{array}{llllll}\text { Яваад } & \text { очсон } & \text { чинь } & \text { Дорж } & \text { сууж байна. } \\ \text { yav-ād } & \text { oč-son } & \text { čin' } & \text { Dorž } & \text { sū-ž } & \text { bai-na. } \\ \text { go-CP } & \text { go.there-NP } & \text { PTC } & \text { Dorj } & \text { sit-CI be-IMPRF }\end{array}$

'I arrived there and, oops, Dorj was sitting there.'

$\begin{array}{lllll}\text { Гэрт } & \text { нь } & \text { ява丿д } & \text { очиж⿻ } & \text { зурхэлсэнгуй } \\ \text { ger-t } & \text { n' } & \text { yav-ād } & \text { oči-ž } & \text { zürxel-sen-güi. } \\ \text { home-DL } & \text { POSS3SG } & \text { go-CP } & \text { go.there-CI } & \text { dare-NP-NEG }\end{array}$

'He did not dare to go directly to her place.'

\subsubsection{The verb yav-in final phrasal position}

When used as a final component of verbal phrases, the verb yav-often expresses a spatial meaning of motion directed away from the location of the speaker, or from the scene of the action.
(41) ДYY
чинь
өчигдөр
ирээд
явсан.
dü
čin'
öčigdör
ir-ēd
yav-san.
younger.sibling POSS2SG yesterday
come-CP
go-NP
'Your brother came around yesterday.'

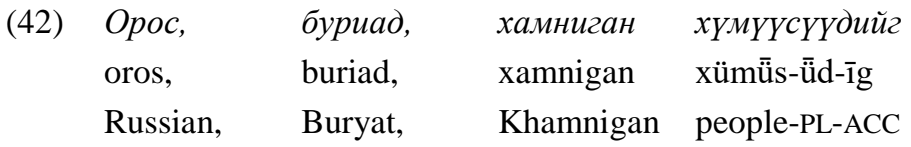




$\begin{array}{llll}\text { зэвсгийн } & \text { хүчээр } & \text { аваад } & \text { явсан. } \\ \text { zevsg-īn } & \text { хӥс̌-е̄r } & \text { av-ād } & \text { yav-san. } \\ \text { arm-GEN } & \text { power-INS } & \text { take-CP } & \text { go-PERF }\end{array}$

'By using the might of weapons, he took away the Russian, Buryat and Khamnigan people.'

$\begin{array}{llllll}\text { Сурч } & \text { байсан } & \text { сургуулиас } & \text { нь } & \text { aваaд } \\ \text { sur-č } & \text { bai-san } & \text { surgūl'-ās } & \text { n' } & \text { av-ād } & \text { yav-san. } \\ \text { study-CI } & \text { be-NP } & \text { school-ABL } & \text { POSS3SG } & \text { take-CP } & \text { go-NP }\end{array}$

'They took her out of the school [where] she had been studying.'

(44)

$\begin{array}{lllll}\text { "Дараа } & \text { уулзъя" } & \text { гээд ороод } & \text { явчихсан } & \text { гэнэ. } \\ \text { "darā } & \text { ūlz-ya" } & \text { ge-ed or-ōd } & \text { yav-čix-san } & \text { ge-ne. } \\ \text { later } & \text { meet-VOL } & \text { say-CP enter-CP } & \text { go-INT-NP } & \text { say-IMPRF }\end{array}$

'She said "See you later", entered the house (and disappeared).'

$\begin{array}{lllll}\text { Мафийн } & \text { гишүун } & \text { оросын } & \text { шоронгоос } & \text { халбагаар } \\ \text { Maf-īn } & \text { gišün } & \text { oros-ïn } & \text { šorong-ōs } & \text { xalbag-ār } \\ \text { mafia } & \text { member } & \text { Russian-GEN } & \text { prison-ABL } & \text { spoon-INS }\end{array}$

нүх ухаад гараад явчихаж.

nüx ux-ād gar-ād yav-čixa-ž.

hole dig-CP go.out-CP go-INT-PRET.IMP

'A member of the mafia escaped from a Russian prison [by] digging a hole with his spoon.'

$\begin{array}{llllll}\text { Яаж } & \text { чамайг } & \text { ийм } & \text { хэиҮY } & \text { Үед } & \text { хаяад } \\ \text { yāž } & \text { čama-ig } & \overline{1} \mathrm{~m} & \text { xecü } & \text { üye-d } & \text { xay-ād } \\ \text { how } & \text { 2SG-ACC } & \text { such } & \text { difficult } & \text { time-DL } & \text { throw-CP }\end{array}$

явжс чадав $а$ са.

yav-ž čad-av $\bar{a}$.

go-CI can-PRET.PERF PTC

'How could he abandon you in such a difficult time?'

(47) Манай эндээс морь унаад явсан.

mana-i end-ēs mor' un-ād yav-san.

1PL.GEN here-ABL horse ride-CP go-NP

'He left our place riding [our] horse.' 


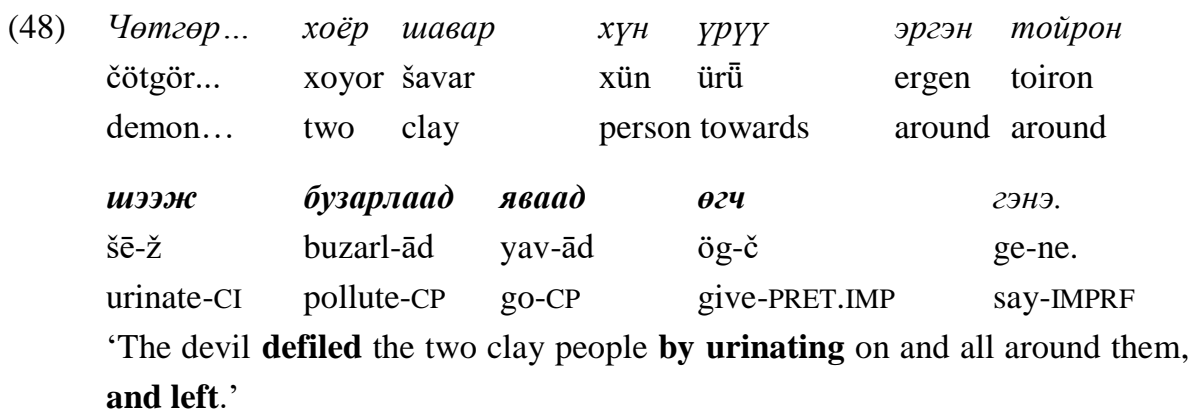

\section{Derived and figurative usages of the verb yav-}

When considering the range of meanings of the Khalkha Mongolian verb yav-, it is nearly impossible to distinguish between basic and derived meanings. The material yields a large portion of examples closely connected with motion; at the same time, the verb yav- may also designate a more general meaning. In some cases, it is best translated with existential verbs (cf. section 3.5).

\subsection{Designation of an activity connected with movement}

The verb yav- is frequently used as a representative expression for an action connected with movement. In these types of phrases, the action is usually specified by a noun - the object or goal of the action - in the dative-locative case.

\begin{tabular}{|c|c|c|c|c|}
\hline Охин & чинь & хичээлдээ & явж & байгаа \\
\hline oxin & čin' & xičēel-d-ēe & yav-̌̌z & baig-ā \\
\hline daughter & 2SG-GEN & class-DL-POSS.REF & go-CI & be-NI \\
\hline
\end{tabular}

'Does your daughter go to school?'

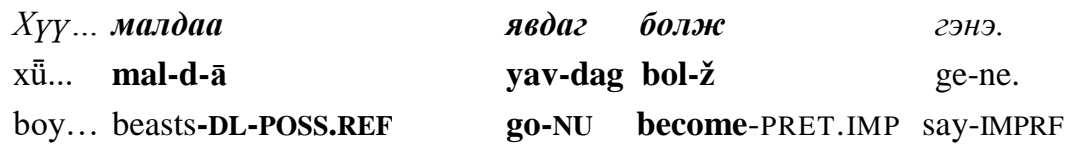

'The boy began herding his beasts regularly.'

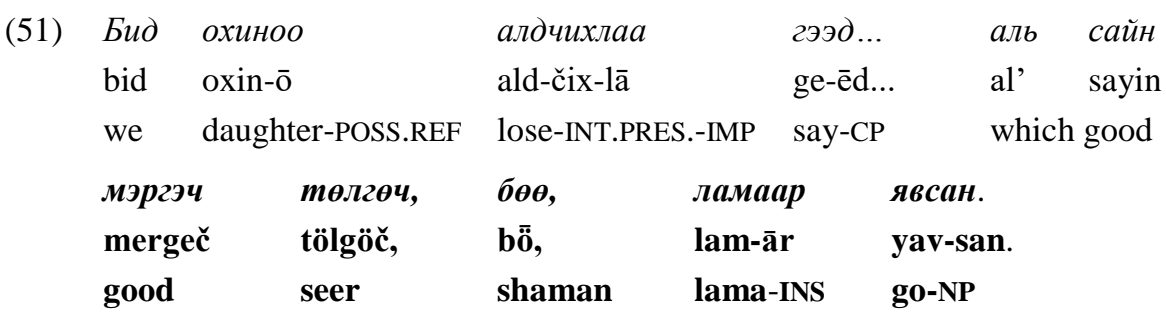


'Since our daughter was missing, we visited every good diviner, shaman and lama.'

$\begin{array}{lllll}\text { Тэгэхэд } & \text { 'Амьд байна. } & \text { Нэг } & \text { хүнтэй } & \text { хамm } \\ \text { tege-xe-d } & \text { 'am'd bai-na. } & \text { neg } & \text { xün-tei } & \begin{array}{l}\text { xamt } \\ \text { do.so-NF-DL }\end{array} \\ \text { alive be-IMPRF } & \text { one } & \text { person-SOC } & \text { together } \\ \text { яваaд } & \text { байнa' } & \text { гэсэн. } & & \\ \text { yav-ād } & \text { bai-na' } & \text { ge-sen. } & & \\ \text { go-CP } & \text { be-IMPRF } & \text { say-NP } & & \end{array}$

'And they said: "She is alive, she is (living, moving) together with another person.",

\subsection{Derivational meanings of 'living, spending time'}

An extremely frequent abstract usage involves instances where the verb yav-is used to refer to life in general, making one's living, success or lack thereof, one's conduct, and so on.

$\begin{array}{lll}\text { Залуудаа } & \text { сайн явсан } & \text { нь } \\ \text { zalū-d-ā } & \text { sain yav-san } & \text { n' } \\ \text { young-DL-POSS } & \text { good go-NP } & \text { POSS3SG } \\ \text { наслахад } & \text { мэдэгдэнэ. } & \\ \text { nasla-Xa-d } & \text { mede-gde-ne. } & \\ \text { grow.old-NF-DL } & \text { know-PAS-IMPRF }\end{array}$

'If you live well in your youth, the results become evident when you grow old.'

$\begin{array}{lllclll}\text { Mуу } & \text { явахад } & \text { нөхөр хол сайн } & \text { явахад } & \text { садан } & \text { ойр. } \\ \text { mū } & \text { yav-ax-ad } & \text { nöхӧr xol } & \text { sain } & \text { yav-ax-ad } & \text { sadan } & \text { oir. } \\ \text { bad } & \text { go-NF-DL } & \text { friend distant good } & \text { go-NF-DL } & \text { relative } & \text { close }\end{array}$
'When things aren't going well, friends are far away; when things are going well, relatives are close by.'

$\begin{array}{lllllll}\text { Үнэнээр } & \boldsymbol{я в с а н ~} & \text { Хүн } & \text { Ухэр } & \text { тэргээр } & \text { туулай } & \text { гүйцุнэ. } \\ \text { ünen-ēr } & \text { yav-san } & \text { xün } & \text { üxer } & \text { terg-ēr } & \text { tūlai } & \text { güic-ne. } \\ \text { truth-INS } & \text { go-NP } & \text { person cow } & \text { cart-INS } & \text { rabbit } & \text { reach }\end{array}$

'Those who live in truth can overtake a rabbit with an oxen cart.' 
(56)

$\begin{array}{lllll}\text { Сохор Тарваа } & \text { ҮҮнээс } & \text { хойш удтал амьд } & \text { явсан. } \\ \text { soxor tarvā } & \text { ün-ēs } & \text { xоǐ̌ udtal am'd } & \text { yav-san. } \\ \text { blind Tarvaa } & \text { this-ABL } & \text { after long alive } & \text { go-NP }\end{array}$

'After that, blind Tarvaa still lived a long time.'

\begin{tabular}{|c|c|c|c|c|}
\hline $\begin{array}{l}\text { ТэнуУн } \\
\text { tenūn } \\
\text { calm }\end{array}$ & $\begin{array}{l}\text { явахад } \\
\text { yava-Xa-d } \\
\text { go-NF-DL }\end{array}$ & $\begin{array}{l}\text { пэмээгээр } \\
\text { temē-gēr } \\
\text { camel-INS }\end{array}$ & $\begin{array}{l}\text { тусалснаас } \\
\text { tusal-san-ās } \\
\text { help-NP-ABL }\end{array}$ & $\begin{array}{l}\text { тэвдэюс } \\
\text { tevde-ž } \\
\text { be at a loss-CI }\end{array}$ \\
\hline & пэвнээр & тусалсан & $H b$ & дээр. \\
\hline xa-d & tevn-ēr & tusal-san & n' & dēr. \\
\hline -DL & awl-INS & help-NP & POSS3SG & above \\
\hline
\end{tabular}

'It is better to help those in need with an awl than to help those who have enough with a camel.'

\begin{tabular}{|c|c|c|c|c|}
\hline 'Хурмаст & \multicolumn{2}{|c|}{ тэнгэрээ } & $\operatorname{maüx}$ & юмсан' \\
\hline 'Xurmast & \multicolumn{2}{|c|}{ tenger- $\overline{\mathrm{e}}$} & tai-x & yumsan' \\
\hline Khurmast & \multicolumn{2}{|c|}{ tengri-ACC-POSS.REF } & worship-NF & PTC \\
\hline гэЖ & бодожс & явдаг & байжээ. & \\
\hline ge- $\check{z}$ & bodo-ž & yav-dag & bai-žè. & \\
\hline ty-CI & think-CI & go-NU & be-PRES.IMP & \\
\hline
\end{tabular}

'He used to think: I would like to make an offering to Khurmast Tengri.'

$\begin{array}{llllll}\text { Ялангуяа } & \text { нэгдлийн } & \text { ажнилд } & \text { их } & \boldsymbol{я в с а н ~} & \text { даa } \\ \text { Yalanguya } & \text { negdel-īn } & \text { ažil-d } & \text { ix } & \text { yav-san } & \text { dā. } \\ \text { above.all } & \text { cooperative-GEN } & \text { work-DL } & \text { much } & \text { go-NP } & \text { PTC }\end{array}$

'I used to work mainly in cooperatives.'

\subsection{Reference to the passage of time}

The phrase yavsār baigād, literally meaning 'having been going [on] for a long time', is idiomatically used to refer to the passage of time. The subject may be either a typical subject of the verb yav- (60) or any other subject (61).

Бид өсөх
bid ösö-x
we grow-N
ээжийнхээ
ěž-īnx-ē

гэж завгүй
ge-ž zavgüi
say-CI busy

$$
\text { явсаap }
$$

байгаад аав

$$
\text { yav-sār }
$$

go-CP

bai-gād

$\bar{a} \mathrm{v}$

$\bar{e} z ̌-i ̄ n x-\bar{e}$

хөгиирч
xögšir-č

байгааг

анзаардаггүй.

bai-gā-g anzār-dag-güi. 
mother-GEN-POSS.REF grow.old-CI be-NI-ACC notice-NU-NEG

'Being busy with [our] growing up, we do not notice how our parents grow old.'

(61)

\begin{tabular}{|c|c|c|c|}
\hline Дүгрэг & гэдэг & $Y^{2} \quad$ явсаар & байгаад \\
\hline dügreg & ge-deg & yav-sār & bai-gād \\
\hline "Dügreg" & say-NU & word go-CA & be-CP \\
\hline төгрөг & болсон & байхаа & \\
\hline tögrög & bol-son & baixā. & \\
\hline “tögrög” & become-NP & probably & \\
\hline
\end{tabular}

\subsection{Figurative usage in place of an existential verb}

The verb yav- may be also idiomatically used for inanimate objects, in which case it is best translated wih an existential verb. Such usage has rich emotional associations, for the most part making the statement more agreeable to the listener.

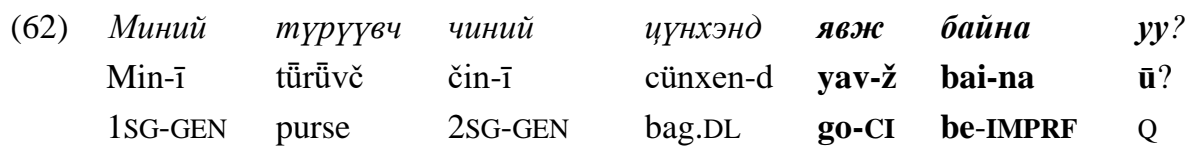

'Is my purse in your bag?'

\subsection{Expression of intention, change of direction}

In modern texts, the figurative usage of the verb yav-, in the sense of 'change of direction, intention', etc. is common. This manner of usage, corresponding to that of European languages and possibly also the result of the influence of the latter, seems nonetheless to conform with the general semantics of the Mongolian verb.

$\begin{array}{llll}\text { (63) Нийгэм } & \text { хаамаа } & \text { яваад } & \text { байна? } \\ \text { n̄̄gem } & \text { xāšā } & \text { yav-ād } & \text { bai-na? } \\ \text { society } & \text { where.to } & \text { go-CP } & \text { be-IMPRF } \\ \text { 'Where is society headed?' } & \end{array}$




\subsection{Metaphor for death}

As in many other languages, the verb yav- is used as a euphemistic expression in reference to death.

$\begin{array}{llll}\text { Жасрайн Жанцан энэ ертөнцөөс гэнэт } & \text { явчихаж. } \\ \text { Žasrain Žancan ene yertönc-ös genet } & \text { yav-čix-až. } \\ \text { Jasrain Jantsan this world-ABL suddenly } & \text { go-INT-PRET } \\ \text { 'Jasrain Jantsan has suddenly left this world.' }\end{array}$

\subsection{Problems in delimitation of ,literal“" and „figurative“" meaning}

Finally, it seems necessary to introduce one important feature of the semantics of the verb yav-. Many instances of usage, which a European linguist would most likely according to the mode of European linguistics - label as figurative, modal, auxiliary, and so on, actually convey the basic and literal meaning of movement. Nonetheless, a semantic overlap with the derivational meanings of existence, living, or the modality of continuous action, still occurs. The following two examples, both taken from folkloric texts, show the verb yav-being used in syntactical or logical parallels in both the literal and basic meaning of motion, along with the figurative meaning of mode of existence, behaviour, habit, and so on.

The first series of examples, taken from the folktale known as Цуут цагаач гүY, ияолмон ияагаан унага (Cūt cagāč gü, colmon cagān unaga), is taken from the section in which the White Mare advises its foal on how to act in his own independent life; the foal subsequently disregards this advice only to then discover the White Mare's reasons behind it. Here, examples of both the literal meaning of movement (67), (69), along with the meaning of habitus or mode of existence (65), (66), (68), (70) accumulate in logical paralells, linked by the image of the life of a Mongolian horse, for whom constant motion is the basic mode of life.

$\begin{array}{llrlll}\text { Айльын } & \text { бууцан } & \text { дээр } & \text { битгий } & \text { унтаж⿻ } & \text { яваарай. } \\ \text { ail-ïn } & \text { būcan } & \text { dēr } & \text { bitgī } & \text { unta-̌̌ } & \text { yav-ārai. } \\ \text { family-GEN } & \text { camping.ground on } & \text { PROH } & \text { sleep-CI } & \text { go-IMP }\end{array}$

'Do not sleep in empty camping grounds.'

\begin{tabular}{|c|c|c|}
\hline ЭрүYл & газар унтажс & \\
\hline erül & gazar unta-̌̌ & \\
\hline healthy & place sleep-CI & \\
\hline
\end{tabular}

'Do sleep in healthy places.' 
(67)

Ижилтэйгээ

явахдаа

alike-SOC-POSS.REF

захад

zapy

zax-ad gar-̌̌

edge-DL

go.out-CI yava-x-d-ā

go-NF-DL-POSS.REF

яваapaй.

yav-ārai.

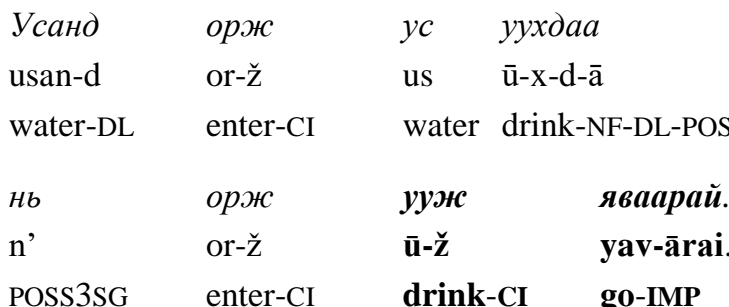

адууньл

adūn- $\ddot{i}$

horse-GEN

'When going to the waterside to drink, be among the first to (step into the water) and drink.'

(69)

\begin{tabular}{|c|c|c|c|c|c|}
\hline Адууньл & дунд & явсан & чинь & июслүУд & $H b$ \\
\hline adūn-ï & dund & yav-san & čin' & iž-lüd & n' \\
\hline herd-GEN & among & go-NP & PTC & alike-PL & POSS3SG \\
\hline ииглөж & алчих & гээд & явуул & сангуй. & \\
\hline šiglö-ž & al-či-x & ge-ēd & yav-ì & l-san-güi. & \\
\hline ck-CI & kill-INT-NF & say-CP & go-C & US-NP-NEG & \\
\hline
\end{tabular}

'While he was running inside the herd, the horses were kicking him, trying to kill him and did not let him run.'

\begin{tabular}{|c|c|c|c|c|c|}
\hline Адууньххаа & & & $H b$ & гараад & явсан \\
\hline adūn-ï-xā & & & n' & gar-ād & yav-san \\
\hline herd-GEN-PO & SS.REF bc & & POSS3SG & go.out-CP & go-NP \\
\hline zmaŭ & сайхан & & & & гэнэ. \\
\hline atai & saixan & & & & ge-ne. \\
\hline easant & nice & & be & ne-PRET.PE & say-IMPRF \\
\hline
\end{tabular}

'As he (learned to) stay to the side of the herd, he finally began enjoying an agreeable life.' 
The next example is from a folk song. The four rhymes display syntactic parallelism, which is a typical device in Mongolian poetry. In the first and the third lines, as a basis of comparison, the verb yav-is used in its more literal meaning of movement, whereas in the second and fourth lines yav-functions as a modal verb: its usage is figurative, referring to certain aspects of life and human behaviour.

$\begin{array}{llll}\text { Уулаар } & \boldsymbol{\text { явдаг }} & \text { угалзын } & \text { зан, } \\ \text { ūl-ār } & \text { yav-dag } & \text { ugalz-ïn } & \text { zan, } \\ \text { mountain-INS } & \text { go-NU } & \text { ibex/muflon-GEN } & \text { character }\end{array}$

'To roam in the mountains is the ibexes' custom,

$\begin{array}{llll}\text { Уулзаад } & \text { явдаг } & \text { хүний } & \text { зан, } \\ \text { ülz-ād } & \text { yav-dag } & \text { хӥn- } \overline{1} & \text { zan, } \\ \text { meet-CP } & \text { go-NU } & \text { person-GEN } & \text { character }\end{array}$

'To live meeting others is a human custom,

$\begin{array}{llll}\text { ҮҮрээр } & \boldsymbol{я в д а г ~} & \text { Үхрийн } & \text { зан, } \\ \text { ür-ēr } & \text { yav-dag } & \text { üxr-īn } & \text { zan, } \\ \text { dawn-INS } & \text { go-NU } & \text { cattle-GEN } & \text { character, }\end{array}$

'To walk at dawn is the habit of cattle,

$\begin{array}{lccc}\text { Үерхэжн } & \boldsymbol{я в д а г ~} & \text { багын } & \text { зан. } \\ \text { üerxe-ž } & \text { yav-dag } & \text { bag-ïn } & \text { zan. } \\ \text { be.friends-CI } & \text { go-NU } & \text { little-GEN } & \text { character. } \\ \text { 'To become close (friends) is the habit of children/youth.' }\end{array}$

\section{Conclusion}

The main semantics of the Khalkha Mongolian verb yav-are connected to motion in the general sense. It refers to motion in space with either no concrete spatial definition (1.1), or with the emphasis on departing from a certain location (1.3). Even when the goal, course and manner of motion are specified in various ways, the emphasis is still placed on the process of motion itself. In more general or abstract usage, the verb yav- often represents the meaning of an entire journey with a specific goal or purpose (1.2). Syntactically, when the spatial goal of motion is expressed, it does not bear the dative-locative case (used mainly to express the purpose of motion), but, instead, the lative case (or postpositional construction), which corroborates the idea of the lack of inherent spatial features of this verb. 
Similarly to other verbs of motion, the verb yav-is used as a component of complex descriptive designations of actions, which is a typical feature of most Altaic languages. The scale of usage options, however, is relatively small, being mostly limited to the expression of one of the basic lexical meanings (2.4.1) or the spatial meaning of motion away from a certain point (2.4.2).

Likewise, the Mongolian verb yav-displays, in comparison to other verbs of motion, relatively few modes of modal usage, which may be the result of its lack of concrete spatial and deictic features. The two main modal meanings of the verb, stemming from its two basic "semantic cores", convey the meaning of durativity or continuity of an action (2.1), and the modality of intense or abrupt change (2.2).

The material used for this study yields examples of usage which could be labelled as auxiliary in the narrower sense (i.e. having predominantly grammatical function) (2.3). This feature, absent in the cases of other verbs of motion, reflects the verb's semantic connection to existence.

In its basic figurative meaning, the Mongolian verb yav-refers to life, existence and its various modes and manners. While, generally speaking, in many cultures, motion is perceived as the representation of life, in the traditional Mongolian nomadic culture, where constant movement is the basic condition of survival, the association of motion with life seems to be even more implicit and essential. Hence, the figurative meaning of the verb yav-covers almost all spheres connected to existence, including staying, dwelling, making one's living, behavior, degree of success in a given endeavor, and so on. The boundaries between the literal and figurative meanings are often difficult to distinguish when these aspects of life happen to overlap with the actual process of motion, as shown in section 3.7.

In my two previous papers (Zikmundová 2009 and 2010) I examined two verbs of motion, whose meaning may be roughly translated as 'to come here' and 'to go there/to visit', in both Khalkha Mongolian and Jungarian Sibe. In both languages these verbs are antonyms and form part of a coherent system of verbs of motion with distinct spatial meanings and deictic functions.

The verb yav-, while being one of the basic verbs of motion, does lack some features usually characteristic of verbs of this system. It does not convey such inherent spatial meaning. Instead, its greater ambiguity with regard to space, as well as its main semantic contours, both resemble the semantics of equivalent verbs in European ("satellite-framed") languages, in particular, the English verb ‘to go'. 
Abbreviations

\begin{tabular}{|c|c|}
\hline $\mathrm{ABL}$ & Ablative \\
\hline ACC & Accusative \\
\hline $\mathrm{CA}$ & Converbum abtemporale \\
\hline CAUS & Causative \\
\hline $\mathrm{CC}$ & Converbum conditionale \\
\hline CI & Converbum imperfecti \\
\hline $\mathrm{CM}$ & Converbum modale \\
\hline $\mathrm{CP}$ & Converbum perfecti \\
\hline CT & Converbum terminale \\
\hline DL & Dative-locative \\
\hline GEN & Genitive \\
\hline IMP & Imperative \\
\hline IMPRF & Imperfective verb \\
\hline INS & Instrumental \\
\hline INT & deverbal suffix of intensity \\
\hline LAT & Lative \\
\hline NEG & Negative \\
\hline NF & Nomen Futuri \\
\hline NI & Nomen Imperfecti \\
\hline NU & Nomen usus \\
\hline PAS & Passive \\
\hline PL & Plural \\
\hline POSS & Possessive \\
\hline POSS.REF & Reflexive possessive suffix \\
\hline PRES.PERF & Presens Perfecti \\
\hline PRET.IMP & Preteritum Imperfecti \\
\hline PRET.PERF & Preteritum Perfecti \\
\hline $\mathrm{PROH}$ & Prohibitive particle \\
\hline PTC & Particle \\
\hline Q & Question marker \\
\hline SOC & Sociative \\
\hline TOP & Topic particle \\
\hline VOC & Vocative \\
\hline VOL & Voluntative \\
\hline VP & Verbum pluritativum \\
\hline
\end{tabular}




\section{References}

Levin, Beth and Rappaport Hovav, Malka 1992. The lexical semantics of verbs of motion: The perspective from unaccusativity. In: Iggy, Rocca (ed.) Thematic structure: its role in grammar. Berlin: Foris. 247-269.

Lubsangdorji, Jugderiin and Vacek, Jaroslav 2004. Colloquial Mongolian: an introductory intensive course. Prague: Triton.

Nakazawa, Tsuneko 2007. A typology of the ground of deictic motion verbs as path conflation verbs: The speaker, the addressee, and beyond. Poznań Studies in Contemporary Linguistics 43/2. 59-82.

Nakazawa, Tsuneko 2009. Typology of the path of deictic motion verbs as path conflation verbs: the entailment of arrival and the deictic center. Poznan Studies in Contemporary Linguistics 45/3. 385-403.

Slobin, Dan I. 1996. Two ways to travel: the verbs of motion in English and Spanish. In: Shibatani and Thompson, S. A. (eds.) Grammatical constructions: their form and meaning. Oxford: Clarendon Press. 195-219.

Talmy, Leonard 1975. Semantics and syntax of motion. In: Kimball, J. P. (ed.) Syntax and semantics 4. New York: Academic Press. 181-238.

Talmy, Leonard 1985. Lexicalization patterns: semantic structure in lexical forms. In: Shopen, T. (ed.) Grammatical categories and the lexicon: Language typology and syntactic description 3. Cambridge: Cambridge University Press. 57-149.

Talmy, Leonard 2000. Toward a Cognitive Semantics. Volume 1: Concept structuring systems. Cambridge, MA: MIT Press.

Word-Allbritton, A. 2004. The Turkmen verb system: Motion, path, manner and figure. IULC Working Papers Online. Indiana University and The University of Alabama - Huntsville. https://www.indiana.edu/ iulcwp/pdfs/04-word.pdf. Zikmundová, Veronika 2010. The function of descriptive verbs in colloquial Sibe 1. The basic pair of verbs of motion ji- 'to come' vs. gen- 'to visit'. MongoloTibetica Pragensia '10, 3/2. Ethnolinguistics, Sociolinguistics. Religion and Culture. Praha: Triton. 95-123.

Zikmundová, V., 2011. The function of descriptive verbs in Khalkha Mongolian 1. The basic pair of verbs of motion ir- 'to come' vs. oč- 'to visit'. MongoloTibetica Pragensia '11, 4/2. Ethnolinguistics, Sociolinguistics, Religion and Culture. Praha: Triton. 
Zikmundová, V. 2013. The Sibe verb yaf-. Mongolo-Tibetica Pragensia '13, 6/1. Linguistics, Ethnolinguistics, Religion and Culture. Praha: Charles University and Triton.

Database of Mongolian folklore texts at http://www.signeta.cz/textsearch/ 